\title{
Capture and Confinement of Solitons in Nonlinear Integrable Systems
}

\author{
V. K. Mel'nikov \\ Laboratory of Theoretical Physics, Joint Institute for Nuclear Research, Head Post Office, P.O. \\ Box 79, SU-101000 Moscow, USSR
}

\begin{abstract}
The system describing the interaction of a long wave with a short wave packet on the $x, y$ plane is considered. The solutions are found to describe a soliton that comes from infinity and then is captured into a conditionally periodical oscillatory regime. The solutions are also found that describe a soliton coming from infinity and then decaying into two solitons: one goes to infinity and the other is captured into a conditionally periodical oscillatory regime. The obtained results are relevant to some problems of hydrodynamics, plasma physics, solid state physics, etc.
\end{abstract}

\section{Introduction}

The present paper is devoted to the problems of capture and confinement of solitons in nonlinear integrable systems. More exactly, the case in point is the following phenomenon. Nonlinear integrable systems considered below have been found to have solutions describing a soliton that comes from infinity, then is captured into a conditionally periodical oscillatory regime and remains in this state in all subsequent times. These systems also have solutions describing a soliton that executed a conditionally periodical oscillatory motion, then came off and went to infinity. Further, we have found solutions describing the soliton that comes from infinity, then decays into two solitons: one being captured into the conditionally periodical oscillatory regime and the other going to infinity. Making a time inversion in these solutions, we get new solutions describing the soliton coming from infinity which collides with the other soliton that has been in the regime of conditionally periodical oscillatory motion; as a result of the collision these two solitons fuse into one soliton that goes to infinity.

We proceed from the following system of equations:

$$
\begin{gathered}
3 \frac{\partial^{2} v}{\partial y^{\prime 2}}-\frac{\partial}{\partial x^{\prime}}\left[\frac{\partial v}{\partial t^{\prime}}+\frac{\partial}{\partial x^{\prime}}\left(3 v^{2}+\frac{\partial^{2} v}{\partial x^{\prime 2}}+8 \kappa|\psi|^{2}\right)\right]=0, \\
i c_{0} \frac{\partial \psi}{\partial t^{\prime}}+i c_{1} \frac{\partial \psi}{\partial x^{\prime}}+i \frac{\partial \psi}{\partial y^{\prime}}=v \psi+\frac{\partial^{2} \psi}{\partial x^{\prime 2}}+c_{2} \frac{\partial^{2} \psi}{\partial y^{\prime 2}}+c_{3}|\psi|^{2} \psi
\end{gathered}
$$


describing the interaction of a long wave with a short wave packet propagating on the $x^{\prime}, y^{\prime}$ plane at an angle to each other. Here $v$ is the long wave amplitude, $\psi$ is the complex short wave envelope, the parameters $c_{0} \neq 0, c_{1}, c_{2}$, and $c_{3}$ take arbitrary real values and $\kappa^{2}=1$. We shall search for a solution of this system in the following form:

$$
\begin{gathered}
v\left(x^{\prime}, y^{\prime}, t^{\prime}\right)=\varepsilon^{2} u\left(\varepsilon x^{\prime}, \varepsilon^{2} y^{\prime}, \varepsilon^{3} t^{\prime}\right), \\
\psi\left(x^{\prime}, y^{\prime}, t^{\prime}\right)=\varepsilon^{2} \varphi\left(\varepsilon x^{\prime}, \varepsilon^{2} y^{\prime}, \varepsilon^{3} t^{\prime}\right) \exp \left[i \sigma\left(x^{\prime}-\tau t^{\prime}\right)\right],
\end{gathered}
$$

where

$$
\sigma=\frac{1}{2} c_{1}, \quad \tau=\frac{1}{2} c_{1} c_{0}^{-1} .
$$

Substituting these expressions into the system (1) we immediately get equations of the form

$$
\begin{aligned}
& 3 \frac{\partial^{2} u}{\partial y^{2}}-\frac{\partial}{\partial x}\left[\frac{\partial u}{\partial t}+\frac{\partial}{\partial x}\left(3 u^{2}+\frac{\partial^{2} u}{\partial x^{2}}+8 \kappa|\varphi|^{2}\right)\right]=0, \\
& i \varepsilon c_{0} \frac{\partial \varphi}{\partial t}+i \frac{\partial \varphi}{\partial y}=u \varphi+\frac{\partial^{2} \varphi}{\partial x^{2}}+\varepsilon^{2} c_{2} \frac{\partial^{2} \varphi}{\partial y^{2}}+\varepsilon^{2} c_{3}|\varphi|^{2} \varphi
\end{aligned}
$$

where $x=\varepsilon x^{\prime}, y=\varepsilon^{2} y^{\prime}$, and $t=\varepsilon^{3} t^{\prime}$. It is naturally expected that at small $\varepsilon \neq 0$ the system (3) can be studied by applying the ideas of perturbation theory. This remark accounts for the importance of investigating the solutions of the system

$$
\begin{gathered}
3 \frac{\partial^{2} u}{\partial y^{2}}-\frac{\partial}{\partial x}\left[\frac{\partial u}{\partial t}+\frac{\partial}{\partial x}\left(3 u^{2}+\frac{\partial^{2} u}{\partial x^{2}}+8 \kappa|\varphi|^{2}\right)\right]=0 \\
i \frac{\partial \varphi}{\partial y}=u \varphi+\frac{\partial^{2} \varphi}{\partial x^{2}}
\end{gathered}
$$

following from (3) at $\varepsilon=0$.

As is known, [1,2] the system (4) can be investigated by the inverse scattering method that was used to detect a great variety of dynamics of soliton solutions of this system [3]. New results obtained along this direction will be given below.

At first, the system (4) has soliton solutions of the form

$$
\begin{gathered}
u=\frac{2 \mu^{2}}{\cosh ^{2}[\mu(x+2 v y-\tau t)]}, \\
\varphi=A \frac{\exp \left[i v x-i\left(\mu^{2}-v^{2}\right) y\right]}{\cosh [\mu(x+2 v y-\tau t)]} \exp (i \sigma t),
\end{gathered}
$$

where the real parameters $\mu, v$, and $\tau$ and the complex quantity $A$ satisfy the only condition

$$
\tau=4\left(\mu^{2}-3 v^{2}+\kappa \mu^{-2}|A|^{2}\right)
$$

and the parameter $\sigma$ takes arbitrary real values. At $A=0$ the soliton (5) degenerates into the well known one-soliton solution [4]

$$
u=\frac{2 \mu^{2}}{\cosh ^{2}\left[\mu x+2 \mu \nu y-4\left(\mu^{2}-3 v^{2}\right) \mu t\right]}, \quad \varphi=0
$$


of the Kadomtsev-Petviashvili equation [5]. Then, at $A \neq 0$ and $\left(\mu^{2}-3 v^{2}\right) \kappa>0$ according to (6), we have $\kappa \tau>0$, i.e., in this case the solitons (5) can propagate only in one direction at any value of the quantity $A$ and fixed values of the parameters $\mu$ and $v$. However, if $A \neq 0$ and $\left(\mu^{2}-3 v^{2}\right) \kappa<0$, the phase velocity $\tau$ of the soliton (5) can take values of both the signs. Thus, in this case depending on the value of the quantity $A$ the solitons (5) can propagate in two opposite directions. Moreover, at $|A|=\left[-\kappa \mu^{2}\left(\mu^{2}-3 v^{2}\right)\right]^{1 / 2}$ we have $\tau=0$, i.e., the soliton (5) is at rest. Below we shall consider the interaction of $N>1$ solitons (5) whose parameters $\mu$ and $v$ are the same and the parameters $\tau=\tau_{m}$ are all different, $m=1, \ldots, N$. It turns out that in this case the $N$-soliton solution of the system (4) obtained by the inverse scattering method describes the evolution of the soliton (5) with the largest value of the quantity $\mu \tau_{m}$ into the soliton with the smallest value of this quantity. In the case $\left(\mu^{2}-3 v^{2}\right) \kappa>0$ the direction of the motion of the initial soliton coincides with that of the final soliton. However, at $\left(\mu^{2}-3 v^{2}\right) \kappa<0$ the direction of the motion of the initial soliton may be opposite to that of the final soliton. Thus, in this case the above-mentioned solution can describe the reflection of the soliton (5). Then, we shall show that $N>1$ solitons (5) with the same values of the parameters $\mu, v$, and $\tau$ but with different values of the parameters $\sigma=\sigma_{m}, m=1, \ldots, N$, produce a solitary wave of the form

$$
\begin{gathered}
u=\frac{2 \mu^{2}}{\cosh ^{2}[\mu(x+2 v y-\tau t-f)]}, \\
\varphi=A \frac{\exp \left[i v x-i\left(\mu^{2}-v^{2}\right) y\right]}{\cosh [\mu(x+2 v y-\tau t-f)]} \exp (i \sigma t),
\end{gathered}
$$

where $f$ and $A$ are $2 \pi$-periodical functions of the variables

$$
\theta_{m, n}=\left(\sigma_{m}-\sigma_{n}\right) t, \quad m, n=1, \ldots, N,
$$

which satisfy the only condition

$$
\frac{d f}{d t}+\tau=4\left(\mu^{2}-3 v^{2}+\kappa \mu^{-2}|A|^{2}\right),
$$

and the frequency $\sigma$ equals one of the quantities $\sigma_{m}$. Hence it follows that the quantity $\tau$ in this case satisfies the condition

$$
\tau=4\left(\mu^{2}-3 v^{2}+\kappa \mu^{-2}\left|A_{0}\right|^{2}\right),
$$

where

$$
\left|A_{0}\right|^{2}=\lim _{T \rightarrow \infty} \frac{1}{T} \int_{0}^{T}|A(t)|^{2} d t .
$$

Thus, the motion of the wave (8) is composed of the uniform motion and conditionally periodical oscillatory motion. In this case $u$-wave conserves its form whereas the amplitude of $\varphi$-wave changes in time as a certain conditionally periodical function $t$. At $\left(\mu^{2}-3 v^{2}\right) \kappa<0$ the case $\tau=0$ is possible. In this situation the solution (8) describes the wave executing as a whole a conditionally periodical oscillatory motion. According to (9)-(11) in this case the relations $|A(t)| \neq\left|A_{0}\right|$ and $\left|A_{0}\right|=\left[-\kappa \mu^{2}\left(\mu^{2}-3 v^{2}\right)\right]^{1 / 2}$ should be fulfilled. We have found the solutions of the 
system (4) which describe the evolution of the soliton (5) into the solitary wave (8) and vice versa - the evolution of the solitary wave (8) into the soliton (5). In a particular case, these solutions describe either the soliton (5) coming from infinity and then captured into a conditionally periodical oscillatory motion or the wave (8) that has executed a conditionally periodical oscillatory motion, then came off and went to infinity as the soliton (5).

Finally note that at $\kappa=1$ the system (4) admits a solution of the form

$$
\begin{gathered}
u=\frac{6 \mu^{2}}{\cosh ^{2}[\mu(x+2 v y-\tau t)]}, \\
\varphi=A \frac{\sinh [\mu(x+2 v y-\tau t)]}{\cosh ^{2}[\mu(x+2 v y-\tau t)]} \exp \left[i v x-i\left(\mu^{2}-v^{2}\right) y+i \sigma t\right],
\end{gathered}
$$

where the real parameters $\mu, v$, and $\tau$ and the complex quantity $A$ satisfy the conditions

$$
\tau=16 \mu^{2}-12 v^{2}, \quad|A|=3 \mu^{2},
$$

and at $\kappa=-1$ the system (4) has the solution

$$
\begin{gathered}
u=\frac{6 \mu^{2}}{\cosh ^{2}[\mu(x+2 v y-\tau t)]}, \\
\varphi=A \frac{\exp \left[i v x-i\left(4 \mu^{2}-v^{2}\right) y\right]}{\cosh ^{2}[\mu(x+2 v y-\tau t)]} \exp (i \sigma t),
\end{gathered}
$$

where the real parameters $\mu, v$, and $\tau$ and the complex quantity $A$ satisfy the conditions

$$
\tau=4\left(\mu^{2}-3 v^{2}\right), \quad|A|=3 \mu^{2} .
$$

We have found the solutions of the system (4) which describe the decays of the waves (12) and (13) into the soliton (7) and the wave (8) and the solutions describing the fusion of the soliton (7) and the wave (8) into one wave (12) or (13). In the case $\left(\mu^{2}-3 v^{2}\right) \kappa<0$ the wave (8) participating in these processes can have $\tau=0$, i.e., can be in a conditionally periodical oscillatory regime.

\section{Particular Case of Multi-Soliton Solution of the System (4)}

Let $N$ be an arbitrary integer satisfying the condition $N>1$. We use a vectorcolumn $\lambda$ with $N+1$ components $\lambda_{m}$ of the form

$$
\lambda_{m}=\left\{\begin{array}{lll}
a_{m} \exp \left[\omega_{1} x-i \omega_{1}^{2} y-4\left(\omega_{1}^{3}+\bar{\varrho}_{m}^{3}\right) t\right], & \text { if } \quad m=1, \ldots, N, \\
a_{m} \exp \left[\omega_{2} x-i \omega_{2}^{2} y-4\left(\omega_{2}^{3}+\bar{\varrho}_{m}^{3}\right) t\right], & \text { if } \quad m=N+1,
\end{array}\right.
$$

where $a_{m} \neq 0$ and $\varrho_{m}$ are complex parameters and quantities $\omega_{1}$ and $\omega_{2}$ admit the representation

$$
\omega_{1}=\mu_{1}+i v, \quad \omega_{2}=\mu_{2}+i v
$$


with real $\mu_{1}, \mu_{2}$, and $v$. Hereafter, the bar above any quantity means complex conjugation. Let then $P$ and $Q$ be square matrices of an order of $N+1$ with the elements

$$
\begin{aligned}
& P_{m, n}=\left\{\begin{array}{lll}
\frac{1}{2 \mu_{1}} \lambda_{m} \bar{\lambda}_{n}, & \text { if } & m, n=1, \ldots, N, \\
\frac{1}{\mu_{1}+\mu_{2}} \lambda_{m} \bar{\lambda}_{n}, & \text { if } & m=N+1 \text { and } n=1, \ldots, N, \\
\frac{1}{2 \mu_{2}} \lambda_{m} \bar{\lambda}_{n}, & \text { if } & m=1, \ldots, N \text { and } n=N+1,
\end{array}\right. \\
& Q_{m, n}=\frac{\kappa}{\varrho_{m}^{3}+\bar{\varrho}_{n}^{3}}, \quad m, n=1, \ldots, N+1 .
\end{aligned}
$$

Assume

$$
D=\operatorname{det}\left|\begin{array}{cc}
\mathbb{1} & -Q \\
P & \mathbb{1}
\end{array}\right|, \quad \Phi=\operatorname{det}\left|\begin{array}{ccc}
0 & 0 & \tilde{e} \\
0 & \mathbb{1} & -Q \\
\lambda & P & \mathbb{1}
\end{array}\right|,
$$

where $\mathbb{1}$ is the unit matrix of an order of $N+1$ and $e$ is the vector-column with $N+1$ components $e_{m}$ equal to unity. Hereafter the tilde " "»" means transposition, i.e., in particular, the transition from the vector-column to the vector-row. According to the results of paper [2] the functions

$$
u=2 \frac{\partial^{2}}{\partial x^{2}} \ln D, \quad \varphi=\frac{\Phi}{D}
$$

satisfy the system (4), i.e., are its solution.

Expressions derived for the multi-soliton solution of the system (4) can be simplified considerably. By virtue of (1.4) the following equalities are valid:

$$
D=\operatorname{det}(\mathbb{1}+P Q), \quad \Phi=\operatorname{det}\left|\begin{array}{cc}
0 & \tilde{e} \\
\lambda & \mathbb{1}+P Q
\end{array}\right| .
$$

Now we take the diagonal matrix $\Lambda=\operatorname{diag}\left(\lambda_{1}, \ldots, \lambda_{N+1}\right)$. From (1.3) we get

$$
\Lambda e=\lambda, \quad \Lambda^{-1} P \bar{\Lambda}^{-1}=\frac{1}{2 \mu_{1}} U, \quad \bar{\Lambda} Q \Lambda=2 \mu_{1} R,
$$

where $U$ and $R$ are the square matrices of an order of $N+1$ with the elements

$$
\begin{gathered}
U_{m, n}=\left\{\begin{array}{lll}
1, & \text { if } & m, n=1, \ldots, N, \\
\frac{2 \mu_{1}}{\mu_{1}+\mu_{2}}, & \text { if } & m=N+1 \text { and } n=1, \ldots, N, \\
\mu_{1} \mu_{2}^{-1}, & \text { if } & m=n=N+1, N \text { and } n=N+1,
\end{array}\right. \\
R_{m, n}=\frac{\kappa \bar{\lambda}_{m} \lambda_{n}}{2\left(\varrho_{m}^{3}+\bar{\varrho}_{n}^{3}\right) \mu_{1}}, \quad m, n=1, \ldots, N+1 .
\end{gathered}
$$


Based on (1.6) and (1.7) there follow the equalities

$$
D=\operatorname{det}(\mathbb{1}+U R), \quad \Phi=\operatorname{det}\left|\begin{array}{cc}
0 & \tilde{\lambda} \\
e & \mathbb{1}+U R
\end{array}\right| .
$$

Let now $H_{0}$ be the orthogonal matrix of an order of $N$ with the elements $H_{m, n}$ such that $H_{N, n}=N^{-1 / 2}, n=1, \ldots, N$. It is obvious that the remaining elements of this matrix satisfy the condition

$$
\sum_{n=1}^{N} H_{m, n}=0, \quad \text { if } \quad 1 \leqq m<N .
$$

Assume

$$
H=\left|\begin{array}{cc}
H_{0} & 0 \\
0 & 1
\end{array}\right|
$$

Then, let $V_{m, n}$ and $S_{m, n}$ be the elements of the matrices $V=H U \tilde{H}$ and $S=H R \tilde{H}$, respectively. Using (1.8), (1.10), and (1.11) one can easily be convinced that all the matrix $V$ elements are equal to zero except for the elements

$$
V_{N, N}=N, \quad V_{N, N+1}=V_{N+1, N}=\frac{2 \mu_{1}}{\mu_{1}+\mu_{2}} N^{1 / 2}, \quad V_{N+1, N+1}=\frac{\mu_{1}}{\mu_{2}},
$$

and analogous elements of the matrix $S$ are equal to

$$
\begin{aligned}
& S_{N, N}=N^{-1} \sum_{m, n=1}^{N} R_{m, n}, \quad S_{N, N+1}=N^{-1 / 2} \sum_{m=1}^{N} R_{m, N+1}, \\
& S_{N+1, N}=N^{-1 / 2} \sum_{n=1}^{N} R_{N+1, n}, \quad S_{N+1, N+1}=R_{N+1, N+1} .
\end{aligned}
$$

According to (1.9) we have

$$
D=\operatorname{det}(\mathbb{1}+V S), \quad \Phi=\operatorname{det}\left|\begin{array}{cc}
0 & \tilde{h} \\
H e & \mathbb{1}+V S
\end{array}\right|,
$$

where $h=H \lambda$ is the vector-column with the components $h_{1}, \ldots, h_{N+1}$. Here

$$
h_{N}=N^{-1 / 2} \sum_{m=1}^{N} \lambda_{m}, \quad h_{N+1}=\lambda_{N+1}
$$

and the components of the vector $H e$ are respectively equal to $0, \ldots, 0, N^{1 / 2}, 1$. Hence, according to (1.12)-(1.15) there follow the equalities

$$
D=\operatorname{det}\left|\begin{array}{cc}
1+W_{1,1} & W_{1,2} \\
W_{2,1} & 1+W_{2,2}
\end{array}\right|, \quad \Phi=\operatorname{det}\left|\begin{array}{ccc}
0 & h_{N} & h_{N+1} \\
N^{1 / 2} & 1+W_{1,1} & W_{1,2} \\
1 & W_{2,1} & 1+W_{2,2}
\end{array}\right|
$$


where

$$
\begin{aligned}
& W_{1,1}=\sum_{m, n=1}^{N} R_{m, n}+\frac{2 \mu_{1}}{\mu_{1}+\mu_{2}} \sum_{n=1}^{N} R_{N+1, n}, \\
& W_{1,2}=N^{1 / 2} \sum_{m=1}^{N} R_{m, N+1}+\frac{2 \mu_{1}}{\mu_{1}+\mu_{2}} N^{1 / 2} R_{N+1, N+1}, \\
& W_{2,1}=\frac{2 \mu_{1}}{\mu_{1}+\mu_{2}} N^{-1 / 2} \sum_{m, n=1}^{N} R_{m, n}+\frac{\mu_{1}}{\mu_{2}} N^{-1 / 2} \sum_{n=1}^{N} R_{N+1, n}, \\
& W_{2,2}=\frac{2 \mu_{1}}{\mu_{1}+\mu_{2}} \sum_{m=1}^{N} R_{m, N+1}+\frac{\mu_{1}}{\mu_{2}} R_{N+1, N+1} .
\end{aligned}
$$

On their basis we get

$$
\begin{gathered}
D=1+\sum_{m, n=1}^{N} R_{m, n}+\frac{\mu_{1}}{\mu_{2}} R_{N+1, N+1} \\
+\frac{\left(\mu_{1}-\mu_{2}\right)^{2} \mu_{1}}{\left(\mu_{1}+\mu_{2}\right)^{2} \mu_{2}} R_{N+1, N+1} \sum_{m, n=1}^{N} R_{m, n} \\
+\frac{2 \mu_{1}}{\mu_{1}+\mu_{2}}\left(\sum_{m=1}^{N} R_{m, N+1}+\sum_{n=1}^{N} R_{N+1, n}\right) \\
-\frac{\left(\mu_{1}-\mu_{2}\right)^{2} \mu_{1}}{\left(\mu_{1}+\mu_{2}\right)^{2} \mu_{2}} \sum_{m, n=1}^{N} R_{m, N+1} R_{N+1, n}, \\
\Phi=-\left\{1-\frac{\mu_{1}-\mu_{2}}{\mu_{1}+\mu_{2}} \sum_{m, n=1}^{N} R_{m, n}-\frac{\left(\mu_{1}-\mu_{2}\right) \mu_{1}}{\left(\mu_{1}+\mu_{2}\right) \mu_{2}} \sum_{n=1}^{N} R_{N+1, n}\right\} \lambda_{N+1} \\
-\left\{1+\frac{\mu_{1}-\mu_{2}}{\mu_{1}+\mu_{2}} \sum_{m=1}^{N} R_{m, N+1}+\frac{\left(\mu_{1}-\mu_{2}\right) \mu_{1}}{\left(\mu_{1}+\mu_{2}\right) \mu_{2}} R_{N+1, N+1}\right\} \sum_{m=1}^{N} \lambda_{m} .
\end{gathered}
$$

Now assume

$$
\varrho_{m}^{3}=\left\{\begin{array}{lll}
-\bar{\omega}_{1}^{3}+\frac{1}{4}\left(\mu_{1} \tau_{m}+i \sigma_{m}\right), & \text { if } & m=1, \ldots, N \\
-\bar{\omega}_{2}^{3}+\frac{1}{4}\left(\mu_{2} \tau_{m}+i \sigma_{m}\right), & \text { if } & m=N+1
\end{array}\right.
$$

where $\sigma_{m}$ and $\tau_{m}$ are the real parameters. As a result, the components $\lambda_{m}$ of the vector-column $\lambda$ admit the representation

$$
\lambda_{m}= \begin{cases}\zeta_{m} \exp \left[\mu_{1}\left(x+2 v y-\tau_{m} t\right)\right], & \text { if } \quad m=1, \ldots, N, \\ \zeta_{m} \exp \left[\mu_{2}\left(x+2 v y-\tau_{m} t\right)\right], & \text { if } \quad m=N+1,\end{cases}
$$

where according to (1.1), (1.2), and (1.18) the components $\zeta_{m}$ of the vector-column $\zeta$ have the form

$$
\zeta_{m}=\left\{\begin{array}{lll}
a_{m} \exp \left[i v x-i\left(\mu_{1}^{2}-v^{2}\right) y+i \sigma_{m} t\right], & \text { if } & m=1, \ldots, N \\
a_{m} \exp \left[i v x-i\left(\mu_{2}^{2}-v^{2}\right) y+i \sigma_{m} t\right], & \text { if } & m=N+1
\end{array}\right.
$$


Then, on the basis of (1.8), (1.18), and (1.19) we get that at $m, n=1, \ldots, N$ the following equalities are valid:

$$
\begin{gathered}
R_{m, n}=\frac{2 \kappa \bar{\zeta}_{m} \zeta_{n} \exp \left[2 \mu_{1}(x+2 v y)-\left(\tau_{m}+\tau_{n}\right) \mu_{1} t\right]}{\left(\tau_{m}+\tau_{n}-8 \mu_{1}^{2}+24 v^{2}\right) \mu_{1}^{2}+i\left(\sigma_{m}-\sigma_{n}\right) \mu_{1}}, \\
R_{m, N+1}=\frac{2 \kappa \bar{\zeta}_{m} \zeta_{N+1} \exp \left[\left(\mu_{1}+\mu_{2}\right)(x+2 v y)-\left(\mu_{1} \tau_{m}+\mu_{2} \tau_{N+1}\right) t\right]}{\left(\tau_{m}-4 \mu_{1}^{2}+12 v^{2}\right) \mu_{1}^{2}+\left(\tau_{N+1}-4 \mu_{2}^{2}+12 v^{2}\right) \mu_{1} \mu_{2}+i \gamma_{m}} \\
R_{N+1, n}=\frac{2 \kappa \zeta_{n} \bar{\zeta}_{N+1} \exp \left[\left(\mu_{1}+\mu_{2}\right)(x+2 v y)-\left(\mu_{1} \tau_{n}+\mu_{2} \tau_{N+1}\right) t\right]}{\left(\tau_{n}-4 \mu_{1}^{2}+12 v^{2}\right) \mu_{1}^{2}+\left(\tau_{N+1}-4 \mu_{2}^{2}+12 v^{2}\right) \mu_{1} \mu_{2}-i \gamma_{n}}
\end{gathered}
$$

where

$$
\gamma_{m}=\left(\sigma_{m}-\sigma_{N+1}\right) \mu_{1}+12\left(\mu_{1}^{2}-\mu_{2}^{2}\right) \mu_{1} v, \quad m=1, \ldots, N
$$

and at $m=n=N+1$ we have

$$
R_{N+1, N+1}=\frac{\kappa\left|\zeta_{N+1}\right|^{2} \exp \left[2 \mu_{2}\left(x+2 v y-\tau_{N+1} t\right)\right]}{\left(\tau_{N+1}-4 \mu_{2}^{2}+12 v^{2}\right) \mu_{1} \mu_{2}} .
$$

\section{Capture and Confinement of Solitons in the System (4)}

First, let us consider the case with $\mu_{1}=\mu_{2}=\mu$. By virtue of (1.16) and (1.17) we get that in this case the following equalities hold:

$$
D=1+\sum_{m, n=1}^{N+1} R_{m, n}, \quad \Phi=-\sum_{m=1}^{N+1} \lambda_{m}
$$

where according to (1.19)-(1.24) at $m, n=1, \ldots, N+1$, we have

$$
\begin{aligned}
R_{m, n} & =\frac{2 \kappa \bar{\eta}_{m} \eta_{n} \exp \left[2 \mu(x+2 v y)-\left(\tau_{m}+\tau_{n}\right) \mu t\right]}{\left(\tau_{m}+\tau_{n}-8 \mu^{2}+24 v^{2}\right) \mu^{2}+i\left(\sigma_{m}-\sigma_{n}\right) \mu}, \\
\lambda_{m} & =\eta_{m} \exp \left[\mu\left(x+2 v y-\tau_{m} t\right)\right] \exp \left[i v x-i\left(\mu^{2}-v^{2}\right) y\right], \\
\eta_{m} & =a_{m} \exp \left(i \sigma_{m} t\right) .
\end{aligned}
$$

Let us elucidate the behaviour of the solution (1.5) of the system (4) under such a choice of the functions $D$ and $\Phi$. For this purpose we assume that the quantities $\alpha_{m}=\mu \tau_{m}+i \sigma_{m}$ are all different, $m=1, \ldots, N$. Then assume that at $m=1, \ldots, N+1$ the inequality

$$
\left(\tau_{m}-4 \mu^{2}+12 v^{2}\right) \kappa>0
$$

holds. Obviously, the functions $D$ and $\Phi$ admit the representation

$$
\begin{aligned}
& D=1+K \exp [2 \mu(x+2 v y)], \\
& \Phi=L \exp [\mu(x+2 v y)] \exp \left[i v x-i\left(\mu^{2}-v^{2}\right) y\right],
\end{aligned}
$$


where according to (2.1) and (2.2) we have

$$
\begin{aligned}
K & =2 \kappa \sum_{m, n=1}^{N+1} \frac{\bar{a}_{m} a_{n} \exp \left[-\left(\mu \tau_{m}+i \sigma_{m}\right) t\right] \exp \left[-\left(\mu \tau_{n}-i \sigma_{n}\right) t\right]}{\left(\tau_{m}+\tau_{n}-8 \mu^{2}+24 v^{2}\right) \mu^{2}+i\left(\sigma_{m}-\sigma_{n}\right) \mu}, \\
L & =-\sum_{m=1}^{N+1} a_{m} \exp \left[-\left(\mu \tau_{m}-i \sigma_{m}\right) t\right]
\end{aligned}
$$

and consequently, the functions $K$ and $L$ depend only on $t$. With the aforementioned assumptions taken into account the quadratic form $K$ is positive definite. This means that $K>0$ at any real value of $t$. Using (1.5) and (2.4) we get that the solution we are interested in has the form

$$
\begin{aligned}
& u=\frac{2 \mu^{2}}{\cosh ^{2}[\mu(x+2 v y-f)]}, \\
& \varphi=A \frac{\exp \left[i v x-i\left(\mu^{2}-v^{2}\right) y\right]}{\cosh [\mu(x+2 v y-f)]},
\end{aligned}
$$

where

$$
f=-\frac{1}{2 \mu} \ln K, \quad A=\frac{1}{2} L K^{-1 / 2}
$$

Based on (2.5) one can easily be convinced that the relation

$$
\mu \frac{d K}{d t}+8\left(\mu^{2}-3 v^{2}\right) \mu^{2} K+2 \kappa|L|^{2}=0
$$

is valid. Hence it follows that the functions $f$ and $A$ determined by (2.7) satisfy the relation

$$
\frac{d f}{d t}=4\left(\mu^{2}-3 v^{2}+\kappa \mu^{-2}|A|^{2}\right) .
$$

It is to be mentioned that the functions $u$ and $\varphi$ of the form (2.6) always satisfy the system (4) if the functions $f$ and $A$ entering into (2.6) satisfy the relation (2.8). Thus, using an arbitrary complex function $A(t)$ and assuming

$$
f=f_{0}+4 \int_{t_{0}}^{t}\left(\mu^{2}-3 v^{2}+\kappa \mu^{-2}|A(\tau)|^{2}\right) d \tau,
$$

we always get a solution of the system (4) in the form (2.6). However, not under any choice of the function $A(t)$ this solution can be derived from the multi-soliton solution of the system (4).

Now let us elucidate the dynamics of the solution (2.6) under the above choice of the functions $f$ and $A$. For this purpose we use integers $N_{-}$and $N_{+}$such that $1 \leqq N_{-}<N_{+} \leqq N+1$ and assume that $\tau_{1}=\ldots=\tau_{N_{-}}$and $\tau_{N_{+}}=\ldots=\tau_{N+1}$. Further we assume that at $m=N_{-}+1, \ldots, N+1$, the inequality

$$
\left(\tau_{1}-\tau_{m}\right) \mu>0, \quad m=N_{-}+1, \ldots, N+1,
$$


is valid and at $m=1, \ldots, N_{+}-1$ the inequality

$$
\left(\tau_{m}-\tau_{N+1}\right) \mu>0, \quad m=1, \ldots, N_{+}-1,
$$

is valid. Now assume

$$
\begin{gathered}
K_{-}=\sum_{m, n=1}^{N} \frac{2 \kappa \bar{\eta}_{m} \eta_{n}}{\left(\tau_{m}+\tau_{n}-8 \mu^{2}+24 v^{2}\right) \mu^{2}+i\left(\sigma_{m}-\sigma_{n}\right) \mu}, \\
K_{+}=\sum_{m, n=N_{+}}^{N+1} \frac{2 \kappa \bar{\eta}_{m} \eta_{n}}{\left(\tau_{m}+\tau_{n}-8 \mu^{2}+24 v^{2}\right) \mu^{2}+i\left(\sigma_{m}-\sigma_{n}\right) \mu}, \\
L_{-}=-\sum_{m=1}^{N-} \eta_{m}, \quad L_{+}=-\sum_{m=N_{+}}^{N+1} \eta_{m}, \quad \eta_{m}=a_{m} \exp \left(i \sigma_{m} t\right) .
\end{gathered}
$$

Using (2.9) and (2.10) one can easily be convinced that in this situation our solution (2.6) of the system (4) as $t \rightarrow-\infty$ has the asymptotics

$$
\begin{gathered}
u \sim \frac{2 \mu^{2}}{\cosh ^{2}\left[\mu\left(x+2 v y-\tau_{1} t-q_{-}\right)\right]}, \\
\varphi \sim A_{-} \frac{\exp \left[i v x-i\left(\mu^{2}-v^{2}\right) y\right]}{\cosh \left[\mu\left(x+2 v y-\tau_{1} t-q_{-}\right)\right]},
\end{gathered}
$$

where

$$
q_{-}=-\frac{1}{2 \mu} \ln K_{-}, \quad A_{-}=\frac{1}{2} L_{-} K_{-}^{-1 / 2},
$$

and as $t \rightarrow \infty$ this solution has the asymptotics

$$
\begin{gathered}
u \sim \frac{2 \mu^{2}}{\cosh ^{2}\left[\mu\left(x+2 v y-\tau_{N+1} t-q_{+}\right)\right]}, \\
\varphi \sim A_{+} \frac{\exp \left[i v x-i\left(\mu^{2}-v^{2}\right) y\right]}{\cosh \left[\mu\left(x+2 v y-\tau_{N+1} t-q_{+}\right)\right]},
\end{gathered}
$$

where

$$
q_{+}=-\frac{1}{2 \mu} \ln K_{+}, \quad A_{+}=\frac{1}{2} L_{+} K_{+}^{-1 / 2} .
$$

Thus, our solution of the system (4) describes the evolution of the wave (2.12) into the wave (2.14). In the general picture of this phenomenon we should like to emphasize several important particular cases. First, at $N_{-}>1$ and $N_{+}<N+1$ the solution (2.6) according to (2.11)-(2.15) describes the evolution of the wave of the form (8) into the wave of the same form. Then at $N_{-}=1$ and $N_{+}<N+1$ our solution describes the evolution of the soliton (5) into the wave of the form (8), and at $N_{-}>1$ and $N_{+}=N+1$ this solution describes the evolution of the wave of the form (8) into the soliton (5). Finally, at $N_{-}=1$ and $N_{+}=N+1$ our solution describes the evolution of the soliton (5) with the largest value of the phase velocity into the soliton with the smallest value of the phase velocity. Moreover, it is to be mentioned that at $\left(\mu^{2}-3 v^{2}\right) \kappa<0$ according to (2.3) both the cases $\tau_{1}=0$ and $\tau_{N+1}=0$ are possible. In this situation at $N_{-}=1, N_{+}<N+1$, and $\tau_{N+1}=0$ our solution of the system (4) describes the capture of the soliton (5) into the oscillatory 
regime. On the contrary, if $\tau_{1}=0$, then at $N_{-}>1$ and $N_{+}=N+1$ our solution describes the wave of the form (8) which has executed the oscillatory motion, then came off and went to infinity in the form of the soliton (5).

\section{Decay and Fusion of Solitons in the System (4)}

Now we are able to get the solutions of the system (4) describing the decays of the waves (12) and (13) into the soliton (7) and the wave (8), and the solutions describing the fusion of the soliton (7) and the wave (8) into one wave (12) or (13). For this purpose we put in the equalities (1.18)-(1.24) that

$$
a_{N+1}=\varepsilon a, \quad \tau_{N}=\tau_{N+1}=4 \mu_{2}^{2}-12 v^{2}+\varepsilon^{2} \kappa c
$$

and pass to the limit as $\varepsilon \rightarrow 0$. As a result, we get that at $m, n=1, \ldots, N-1$ the following equalities are valid:

$$
\begin{aligned}
& R_{m, n}=\frac{2 \kappa \bar{\eta}_{m} \eta_{n} \exp \left[2 \mu_{1}(x+2 v y)-\left(\tau_{m}+\tau_{n}\right) \mu_{1} t\right]}{\left(\tau_{m}+\tau_{n}-8 \mu_{1}^{2}+24 v^{2}\right) \mu_{1}^{2}+i\left(\sigma_{m}-\sigma_{n}\right) \mu_{1}}, \\
& R_{m, N}=\frac{2 \kappa \bar{\eta}_{m} \eta_{N} \exp \left[2 \mu_{1}(x+2 v y)-\left(\tau_{m}+4 \mu_{2}^{2}-12 v^{2}\right) \mu_{1} t\right]}{\left(\tau_{m}+4 \mu_{2}^{2}-8 \mu_{1}^{2}+12 v^{2}\right) \mu_{1}^{2}+i\left(\sigma_{m}-\sigma_{N}\right) \mu_{1}}, \\
& R_{N, n}=\frac{2 \kappa \eta_{n} \bar{\eta}_{N} \exp \left[2 \mu_{1}(x+2 v y)-\left(\tau_{n}+4 \mu_{2}^{2}-12 v^{2}\right) \mu_{1} t\right]}{\left(\tau_{n}+4 \mu_{2}^{2}-8 \mu_{1}^{2}+12 v^{2}\right) \mu_{1}^{2}-i\left(\sigma_{n}-\sigma_{N}\right) \mu_{1}},
\end{aligned}
$$

where at $m=1, \ldots, N$ we have

$$
\eta_{m}=a_{m} \exp \left(i \sigma_{m} t\right)
$$

Then the equalities

$$
\begin{aligned}
R_{N, N} & =\frac{\kappa\left|a_{N}\right|^{2} \exp \left[2 \mu_{1}(x+2 v y)-2 \mu_{1}\left(4 \mu_{2}^{2}-12 v^{2}\right) t\right]}{4\left(\mu_{2}^{2}-\mu_{1}^{2}\right) \mu_{1}^{2}}, \\
R_{N+1, N+1} & =\frac{|a|^{2}}{c \mu_{1} \mu_{2}} \exp \left[2 \mu_{2}(x+2 v y)-2 \mu_{2}\left(4 \mu_{2}^{2}-12 v^{2}\right) t\right]
\end{aligned}
$$

hold. Finally, by virtue of (3.1) we have $\lambda_{N+1}=0$, and consequently, at any $m, n=1, \ldots, N$ we get

$$
R_{m, N+1}=R_{N+1, n}=0 .
$$

With these equalities taken into account expressions (1.16) and (1.17) acquire the form

$$
\begin{aligned}
& D=1+\sum_{m, n=1}^{N} R_{m, n}+\frac{\mu_{1}}{\mu_{2}} R_{N+1, N+1} \\
&+\frac{\left(\mu_{1}-\mu_{2}\right)^{2} \mu_{1}}{\left(\mu_{1}+\mu_{2}\right)^{2} \mu_{2}} R_{N+1, N+1} \sum_{m, n=1}^{N} R_{m, n}, \\
& \Phi=-\left\{1+\frac{\left(\mu_{1}-\mu_{2}\right) \mu_{1}}{\left(\mu_{1}+\mu_{2}\right) \mu_{2}} R_{N+1, N+1}\right\} \exp \left[i v x-i\left(\mu_{1}^{2}-v^{2}\right) y\right] \\
& \times \sum_{m=1}^{N} \eta_{m} \exp \left[\mu_{1}\left(x+2 v y-\tau_{m} t\right)\right] .
\end{aligned}
$$


Let us now analyse the behaviour of the solution (1.5) of the system (4) under the above-mentioned choice of the functions $D$ and $\Phi$. For this purpose we assume that at $m=1, \ldots, N$ the inequalities

$$
\left(\tau_{m}-4 \mu_{1}^{2}+12 v^{2}\right) \kappa>0, \quad \tau_{N}=\tau_{N+1}=4 \mu_{2}^{2}-12 v^{2},
$$

are valid. Assume then that the conditions

$$
\mu_{1} \mu_{2}>0, \quad c>0
$$

hold. Based on these inequalities according to (3.2)-(3.6) we get that at any real values of the coordinates $x, y$, and $t$ the inequality $D \geqq 1$ is valid. Hence, it follows that the solution of the system (4) determined by (1.5) has no singularities at any real values of independent variables $x, y$, and $t$. Assume finally that at $m=1, \ldots, N-1$ the inequality

$$
\left(4 \mu_{2}^{2}-12 v^{2}-\tau_{m}\right) \mu_{1}>0
$$

is valid.

Now we use arbitrary $\tau \in(-\infty, \infty)$ and assume $z=x+2 v y-\tau t$. According to (3.2), (3.3), and (3.5) expressions for nonzero elements $R_{m, n}$ of the matrix $R$ become

$$
\begin{gathered}
R_{m, n}=\frac{2 \kappa \bar{\eta}_{m} \eta_{n} \exp \left[\left(2 \tau-\tau_{m}-\tau_{n}\right) \mu_{1} t\right] \exp \left(2 \mu_{1} z\right)}{\left(\tau_{m}+\tau_{n}-8 \mu_{1}^{2}+24 v^{2}\right) \mu_{1}^{2}+i\left(\sigma_{m}-\sigma_{n}\right) \mu_{1}}, \\
R_{m, N}=\frac{2 \kappa \bar{\eta}_{m} \eta_{N} \exp \left[\left(2 \tau-\tau_{m}-4 \mu_{2}^{2}+12 v^{2}\right) \mu_{1} t\right]}{\left(\tau_{m}+4 \mu_{2}^{2}-8 \mu_{1}^{2}+12 v^{2}\right) \mu_{1}^{2}+i\left(\sigma_{m}-\sigma_{N}\right) \mu_{1}} \exp \left(2 \mu_{1} z\right), \\
R_{N, n}=\frac{2 \kappa \eta_{n} \bar{\eta}_{N} \exp \left[\left(2 \tau-\tau_{n}-4 \mu_{2}^{2}+12 v^{2}\right) \mu_{1} t\right]}{\left(\tau_{n}+4 \mu_{2}^{2}-8 \mu_{1}^{2}+12 v^{2}\right) \mu_{1}^{2}-i\left(\sigma_{n}-\sigma_{N}\right) \mu_{1}} \exp \left(2 \mu_{1} z\right)
\end{gathered}
$$

if $m, n=1, \ldots, N-1$ and

$$
\begin{array}{r}
R_{N, N}=\frac{\kappa\left|a_{N}\right|^{2} \exp \left(2 \mu_{1} z\right)}{4\left(\mu_{2}^{2}-\mu_{1}^{2}\right) \mu_{1}^{2}} \exp \left[2 \mu_{1}\left(\tau-4 \mu_{2}^{2}+12 v^{2}\right) t\right], \\
R_{N+1, N+1}=\frac{|a|^{2}}{c \mu_{1} \mu_{2}} \exp \left(2 \mu_{2} z\right) \exp \left[2 \mu_{2}\left(\tau-4 \mu_{2}^{2}+12 v^{2}\right) t\right] .
\end{array}
$$

Assume first that the quantity $\tau$ is chosen so that the inequality

$$
\left(\tau-4 \mu_{2}^{2}+12 v^{2}\right) \mu_{1}>0
$$

holds. It results, on the basis of (3.10), in the inequality $\left(\tau-\tau_{m}\right) \mu_{1}>0, m=1, \ldots, N$. By virtue of (3.6), (3.7), and (3.11)-(3.13) it follows that at any fixed $z$ and $t \rightarrow-\infty$ the asymptotics $D \rightarrow 1$ and $\Phi \rightarrow 0$ are valid. With (1.5) taken into account this means that at any fixed $z$ and $t \rightarrow-\infty$ we have $u \rightarrow 0$ and $\varphi \rightarrow 0$. Thus, our solution of the system (4) as $t \rightarrow-\infty$ contains no moving waves with the phase velocity $\tau$ satisfying the condition (3.14). Then assume that the quantity $\tau$ is chosen so as to fulfil the inequality

$$
\left(\tau-4 \mu_{2}^{2}+12 v^{2}\right) \mu_{1}<0 .
$$


Then, according to (3.6), (3.7), and (3.9)-(3.13) at any fixed $z$ and $t \rightarrow-\infty$, the following asymptotics are valid:

$$
\begin{aligned}
& D \exp \left[-2\left(\mu_{1}+\mu_{2}\right)\left(\tau-4 \mu_{2}^{2}+12 v^{2}\right) t\right] \\
& \sim-\frac{\kappa|a|^{2}\left|a_{N}\right|^{2}}{4 c \mu_{1}^{2} \mu_{2}^{2}} \frac{\mu_{1}-\mu_{2}}{\left(\mu_{1}+\mu_{2}\right)^{3}} \exp \left[2\left(\mu_{1}+\mu_{2}\right) z\right], \\
& \Phi \exp \left[-2\left(\mu_{1}+\mu_{2}\right)\left(\tau-4 \mu_{2}^{2}+12 v^{2}\right) t\right] \rightarrow 0 .
\end{aligned}
$$

Based on (1.5) it follows that at any fixed $z$ and $t \rightarrow-\infty$ the relations $u \rightarrow 0$ and $\varphi \rightarrow 0$ hold. Thus, according to (3.14) and (3.15) we get that our solution of the system (4) as $t \rightarrow-\infty$ has no moving waves with the phase velocity $\tau \neq 4 \mu_{2}^{2}-12 v^{2}$. Assume finally that $\tau=4 \mu_{2}^{2}-12 v^{2}$. In this case, on the basis of (3.6), (3.7), and (3.10)-(3.13) we find that at any fixed $z$ and $t \rightarrow-\infty$, the following asymptotics are valid:

$$
\begin{aligned}
D \sim 1 & +R_{N, N}+\frac{\mu_{1}}{\mu_{2}} R_{N+1, N+1} \\
& +\frac{\left(\mu_{1}-\mu_{2}\right)^{2} \mu_{1}}{\left(\mu_{1}+\mu_{2}\right)^{2} \mu_{2}} R_{N, N} R_{N+1, N+1}, \\
\Phi \sim & -\left\{1+\frac{\left(\mu_{1}-\mu_{2}\right) \mu_{1}}{\left(\mu_{1}+\mu_{2}\right) \mu_{2}} R_{N+1, N+1}\right\} \eta_{N} \exp \left(\mu_{1} z\right) \\
& \times \exp \left[i v x-i\left(\mu_{1}^{2}-v^{2}\right) y\right] .
\end{aligned}
$$

In this case

$$
\begin{aligned}
R_{N, N} & =\frac{\kappa\left|a_{N}\right|^{2} \exp \left(2 \mu_{1} z\right)}{4\left(\mu_{2}^{2}-\mu_{1}^{2}\right) \mu_{1}^{2}}, \\
R_{N+1, N+1} & =\frac{|a|^{2}}{c \mu_{1} \mu_{2}} \exp \left(2 \mu_{2} z\right) .
\end{aligned}
$$

Let $\kappa=1$. Assume $\mu_{1}=\mu$ and $\mu_{2}=2 \mu$. Then, by virtue of (3.16) and (3.17), we get that at any fixed $z$ and $t \rightarrow-\infty$ the asymptotics

$$
\begin{gathered}
D \sim 1+\alpha_{1} \exp (2 \mu z)+\alpha_{2} \exp (4 \mu z)+\alpha_{3} \exp (6 \mu z), \\
\Phi \sim-\left[1-\frac{1}{3} \alpha_{2} \exp (4 \mu z)\right] \eta_{N} \exp (\mu z) \exp \left[i v x-i\left(\mu^{2}-v^{2}\right) y\right]
\end{gathered}
$$

are fulfilled, where

$$
\alpha_{1}=\frac{\left|a_{N}\right|^{2}}{12 \mu^{4}}, \quad \alpha_{2}=\frac{|a|^{2}}{4 c \mu^{2}}, \quad \alpha_{3}=\frac{1}{9} \alpha_{1} \alpha_{2} .
$$

Assume now

$$
c=108 \frac{|a|^{2}}{\left|a_{N}\right|^{4}} \mu^{6},
$$

i.e., we choose $c$ so as to fulfill the condition $3 \alpha_{2}=\alpha_{1}^{2}$. According to (3.18) and (3.19) in this case at any fixed $z$ and $t \rightarrow-\infty$ the asymptotics

$$
\begin{gathered}
D \sim\left[1+\frac{1}{3} \alpha_{1} \exp (2 \mu z)\right]^{3} \\
\Phi \sim-\left[1-\frac{1}{9} \alpha_{1}^{2} \exp (4 \mu z)\right] \eta_{N} \exp (\mu z) \exp \left[i v x-i\left(\mu^{2}-v^{2}\right) y\right]
\end{gathered}
$$


hold. With (1.5) taken into consideration it follows that at $\kappa=1$ and $t \rightarrow-\infty$ our solution contains the only moving wave

$$
\begin{aligned}
u & =\frac{6 \mu^{2}}{\cosh ^{2}\left[\mu(x+2 v y-\tau t)+\delta_{-}\right]}, \\
\varphi & =A \frac{\sinh \left[\mu(x+2 v y-\tau t)+\delta_{-}\right]}{\cosh ^{2}\left[\mu(x+2 v y-\tau t)+\delta_{-}\right]} \exp \left[i v x-i\left(\mu^{2}-v^{2}\right) y+i \sigma t\right],
\end{aligned}
$$

where

$$
\tau=16 \mu^{2}-12 v^{2}, \quad \delta_{-}=\frac{1}{2} \ln \left(\frac{\alpha_{1}}{3}\right), \quad A=-\frac{a_{N}}{2} \exp \left(-\delta_{-}\right), \quad \sigma=\sigma_{N} .
$$

With (3.19) it follows

$$
|A|=3 \mu^{2}
$$

Consider now the case $\kappa=-1$. Let $\mu_{1}=2 \mu$ and $\mu_{2}=\mu$. Using (3.16) and (3.17) we get that in this case at any fixed $z$ and $t \rightarrow-\infty$, the following asymptotics are valid:

$$
\begin{gathered}
D \sim 1+\alpha_{1} \exp (2 \mu z)+\alpha_{2} \exp (4 \mu z)+\alpha_{3} \exp (6 \mu z), \\
\Phi \sim-\left[1+\frac{1}{3} \alpha_{1} \exp (2 \mu z)\right] \eta_{N} \exp (2 \mu z) \exp \left[i v x-i\left(4 \mu^{2}-v^{2}\right) y\right],
\end{gathered}
$$

where

$$
\alpha_{1}=\frac{|a|^{2}}{c \mu^{2}}, \quad \alpha_{2}=\frac{\left|a_{N}\right|^{2}}{48 \mu^{4}}, \quad \alpha_{3}=\frac{1}{9} \alpha_{1} \alpha_{2}
$$

Assume

$$
c=4 \frac{|a|^{2}}{\left|a_{N}\right|}
$$

i.e., we choose $c$ so as to satisfy the condition $\alpha_{1}=\left(3 \alpha_{2}\right)^{1 / 2}$. According to (3.23) and (3.24), in this case at any fixed $z$ and $t \rightarrow-\infty$, the following asymptotics hold:

$$
\begin{gathered}
D \sim\left[1+\left(\frac{1}{3} \alpha_{2}\right)^{1 / 2} \exp (2 \mu z)\right]^{3}, \\
\Phi \sim-\left[1+\left(\frac{1}{3} \alpha_{2}\right)^{1 / 2} \exp (2 \mu z)\right] \eta_{N} \exp (2 \mu z) \exp \left[i v x-i\left(4 \mu^{2}-v^{2}\right) y\right] .
\end{gathered}
$$

With (1.5) it follows that at $\kappa=-1$ and $t \rightarrow-\infty$ our solution has the only moving wave

$$
\begin{aligned}
u & =\frac{6 \mu^{2}}{\cosh ^{2}\left[\mu(x+2 v y-\tau t)+\delta_{-}\right]}, \\
\varphi & =A \frac{\exp \left[i v x-i\left(4 \mu^{2}-v^{2}\right) y\right]}{\cosh ^{2}\left[\mu(x+2 v y-\tau t)+\delta_{-}\right]} \exp (i \sigma t),
\end{aligned}
$$

where

$$
\tau=4 \mu^{2}-12 v^{2}, \quad \delta_{-}=\frac{1}{4} \ln \left(\frac{\alpha_{2}}{3}\right), \quad A=-\frac{\alpha_{N}}{4} \exp \left(-2 \delta_{-}\right), \quad \sigma=\sigma_{N} .
$$

By virtue of (3.24) it follows that the relation (3.22) is valid. 
Let us elucidate now the dynamics of the solution considered here as $t \rightarrow \infty$. We shall assume for definiteness that the integer $N_{0}$ satisfying the condition $0<N_{0}<N$ is such that $\tau_{1}=\ldots=\tau_{N_{0}}$ and at $m=N_{0}+1, \ldots, N$ the inequality

$$
\left(\tau_{1}-\tau_{m}\right) \mu_{1}<0, \quad m=N_{0}+1, \ldots, N,
$$

is valid. Let the quantity $\tau$ be first chosen so that the inequality

$$
\left(\tau-\tau_{1}\right) \mu_{1}<0
$$

is fulfilled. From this inequality, according to (3.27), there follows the inequality $\left(\tau-\tau_{m}\right) \mu_{1}<0, m=1, \ldots, N$. Hence, using (3.6), (3.7), and (3.11)-(3.13) we get that at any fixed $z$ and $t \rightarrow \infty$ the asymptotics $D \rightarrow 1$ and $\Phi \rightarrow 0$ hold. Taking allowance of (1.5) it means that at any fixed $z$ and $t \rightarrow \infty$ the asymptotics $u \rightarrow 0$ and $\varphi \rightarrow 0$ are valid, i.e., our solution of the system (4) as $t \rightarrow \infty$ has no moving waves with the phase velocity $\tau$ satisfying the inequality (3.28). Then, we assume that the quantity $\tau$ is chosen so as to fulfill the condition (3.14) again. According to (3.6), (3.7), (3.9)-(3.13), and (3.27) we get that in this case at any fixed $z$ and $t \rightarrow \infty$ the following asymptotics hold:

$$
\begin{aligned}
& D \exp \left[-2\left(\mu_{1}+\mu_{2}\right)\left(\tau-4 \mu_{2}^{2}+12 v^{2}\right) t\right] \exp \left[-2 \mu_{1}\left(4 \mu_{2}^{2}-12 v^{2}-\tau_{1}\right) t\right] \\
& \sim \frac{\left(\mu_{1}-\mu_{2}\right)^{2}|a|^{2}}{\left(\mu_{1}+\mu_{2}\right)^{2} c \mu_{2}^{2}} K_{0} \exp \left[2\left(\mu_{1}+\mu_{2}\right) z\right], \\
& \Phi \exp \left[-2\left(\mu_{1}+\mu_{2}\right)\left(\tau-4 \mu_{2}^{2}+12 v^{2}\right) t\right] \exp \left[-2 \mu_{1}\left(4 \mu_{2}^{2}-12 v^{2}-\tau_{1}\right) t\right] \rightarrow 0,
\end{aligned}
$$

where

$$
K_{0}=\sum_{m, n=1}^{N_{0}} \frac{2 \kappa \bar{\eta}_{m} \eta_{n}}{2\left(\tau_{1}-4 \mu_{1}^{2}+12 v^{2}\right) \mu_{1}^{2}+i\left(\sigma_{m}-\sigma_{n}\right) \mu_{1}} .
$$

From these asymptotics and equalities (1.5) we easily find that at any fixed $z$ and $t \rightarrow \infty$ the relations $u \rightarrow 0$ and $\varphi \rightarrow 0$ are fulfilled. This means that our solution of the system (4) as $t \rightarrow \infty$ has no moving waves with the phase velocity $\tau$ satisfying the inequality (3.14). Assume finally that the quantity $\tau$ is chosen so that the inequalities

$$
\left(\tau-4 \mu_{2}^{2}+12 v^{2}\right) \mu_{1}<0, \quad\left(\tau-\tau_{1}\right) \mu_{1}>0
$$

are fulfilled simultaneously. Then, by virtue of (3.6), (3.7), (3.11)-(3.13), and (3.27) we get that at any fixed $z$ and $t \rightarrow \infty$ we have

$$
\begin{gathered}
D \exp \left[-2\left(\tau-\tau_{1}\right) \mu_{1} t\right] \sim K_{0} \exp \left(2 \mu_{1} z\right), \\
\Phi \exp \left[-2\left(\tau-\tau_{1}\right) \mu_{1} t\right] \rightarrow 0,
\end{gathered}
$$

where the quantity $K_{0}$ is determined by the equality (3.29). Hence, according to (1.5), it follows that at any fixed $z$ and $t \rightarrow \infty$ the relations $u \rightarrow 0$ and $\varphi \rightarrow 0$ are valid. Thus, based on (3.14), (3.28), and (3.30), we can verify that our solution of the system (4) as $t \rightarrow \infty$ has no moving waves with the phase velocity $\tau$ satisfying the condition

$$
\left(\tau-\tau_{1}\right)\left(\tau-4 \mu_{2}^{2}+12 v^{2}\right) \neq 0 .
$$


On the other hand, at $\tau=4 \mu_{2}^{2}-12 v^{2}$ with the equalities (3.11)-(3.13), we get the representation

$$
\sum_{m, n=1}^{N} R_{m, n}=K \exp \left(2 \mu_{1} z\right)
$$

where the quantity $K$ depends only on $t$ and according to (3.10) we have $K \rightarrow \infty$ as $t \rightarrow \infty$. Hence, on the basis of (3.6) and (3.7), it follows that at any fixed $z$ and $t \rightarrow \infty$ we have

$$
\begin{gathered}
D K^{-1} \exp \left(-2 \mu_{1} z\right) \sim 1+\frac{\left(\mu_{1}-\mu_{2}\right)^{2} \mu_{1}}{\left(\mu_{1}+\mu_{2}\right)^{2} \mu_{2}} R_{N+1, N+1}, \\
\Phi K^{-1} \exp \left(-2 \mu_{1} z\right) \rightarrow 0 .
\end{gathered}
$$

Thus, assuming $\kappa=1, \mu_{1}=\mu, \mu_{2}=2 \mu$ and taking account of (3.13), (3.19), and (3.20) we easily get that as $t \rightarrow \infty$ our solution has a moving wave of the form

$$
u=\frac{8 \mu^{2}}{\cosh ^{2}\left[2 \mu(x+2 v y-\tau t)+\delta_{+}\right]}, \quad \varphi=0,
$$

where

$$
\tau=16 \mu^{2}-12 v^{2}, \quad \delta_{+}=\frac{1}{2} \ln \left(\frac{\alpha_{1}^{2}}{27}\right) .
$$

Then, assuming $\kappa=-1, \mu_{1}=2 \mu$, and $\mu_{2}=\mu$, by virtue of (3.13), (3.24), and (3.25) we get that as $t \rightarrow \infty$ our solution has a moving wave of the form

$$
u=\frac{2 \mu^{2}}{\cosh ^{2}\left[\mu(x+2 v y-\tau t)+\delta_{+}\right]}, \quad \varphi=0,
$$

where

$$
\tau=4 \mu^{2}-12 v^{2}, \quad \delta_{+}=\frac{1}{4} \ln \left(\frac{\alpha_{2}}{27}\right) .
$$

Consider finally the case $\tau=\tau_{1}$. On the basis of equalities (3.6), (3.7), (3.11)-(3.13), and inequality (3.27) we get that at any fixed $z$ and $t \rightarrow \infty$ the following asymptotics are valid:

$$
\begin{gathered}
D \sim 1+\sum_{m, n=1}^{N_{0}} R_{m, n}, \\
\Phi \sim-\exp \left[i v x-i\left(\mu_{1}^{2}-v^{2}\right) y\right] \sum_{m=1}^{N_{0}} \eta_{m} \exp \left(\mu_{1} z\right),
\end{gathered}
$$

i.e.

$$
\begin{gathered}
D \sim 1+K_{0} \exp \left(2 \mu_{1} z\right), \\
\Phi \sim L_{0} \exp \left(\mu_{1} z\right) \exp \left[i v x-i\left(\mu_{1}^{2}-v^{2}\right) y\right],
\end{gathered}
$$

where the quantity $K_{0}$ is determined by the equality (3.29) and

$$
L_{0}=-\sum_{m=1}^{N_{0}} \eta_{m} .
$$


It follows from the equalities (3.29) and (3.34) that the functions $K_{0}$ and $L_{0}$ depends only on $t$. Letting $\kappa=1$ and $\mu_{1}=\mu$ and using (1.5) we easily get that as $t \rightarrow \infty$ our solution has a second moving wave of the form

$$
\begin{aligned}
& u=\frac{2 \mu^{2}}{\cosh ^{2}\left[\mu\left(x+2 v y-\tau_{1} t-q_{0}\right)\right]}, \\
& \varphi=A_{0} \frac{\exp \left[i v x-i\left(\mu^{2}-v^{2}\right) y\right]}{\cosh \left[\mu\left(x+2 v y-\tau_{1} t-q_{0}\right)\right]},
\end{aligned}
$$

where

$$
q_{0}=-\frac{1}{2 \mu} \ln K_{0}, \quad A_{0}=\frac{1}{2} L_{0} K_{0}^{-1 / 2} .
$$

According to (3.29), (3.34), and (3.36) the relation

$$
\frac{d q_{0}}{d t}+\tau_{1}=4\left(\mu^{2}-3 v^{2}+\kappa \mu^{-2}\left|A_{0}\right|^{2}\right)
$$

holds. Then, assuming $\kappa=-1$ and $\mu_{1}=2 \mu$, according to (1.5) we get that as $t \rightarrow \infty$ our solution has the second moving wave

$$
\begin{aligned}
u & =\frac{8 \mu^{2}}{\cosh ^{2}\left[2 \mu\left(x+2 v y-\tau_{1} t-q_{0}\right)\right]}, \\
\varphi & =A_{0} \frac{\exp \left[i v x-i\left(4 \mu^{2}-v^{2}\right) y\right]}{\cosh \left[2 \mu\left(x+2 v y-\tau_{1} t-q_{0}\right)\right]},
\end{aligned}
$$

where

$$
q_{0}=-\frac{1}{4 \mu} \ln K_{0}, \quad A_{0}=\frac{1}{2} L_{0} K_{0}^{-1 / 2} .
$$

By virtue of (3.29), (3.34), and (3.39) we have

$$
\frac{d q_{0}}{d t}+\tau_{1}=16 \mu^{2}-12 v^{2}+\kappa \mu^{-2}\left|A_{0}\right|^{2} .
$$

Taking account of (3.31) we become convinced that we have obtained all moving waves entering into our solution of the system (4) as $t \rightarrow \infty$.

Thus, at $\kappa=1$ our solution describes the decay of the wave (3.21) into the soliton (3.32) and the wave (3.35). According to (3.8) we have $\tau_{N}=16 \mu^{2}-12 v^{2}$ and by virtue of (3.10) the inequality $\left(16 \mu^{2}-12 v^{2}-\tau_{1}\right) \mu>0$ holds. Finally, using (3.37) we get that $\tau_{1}>4 \mu^{2}-12 v^{2}$. It follows from these inequalities that at $\mu>0$ the condition $4 \mu^{2}-12 v^{2}<\tau_{1}<16 \mu^{2}-12 v^{2}$ must hold and at $\mu<0$ the condition $\tau_{1}>16 \mu^{2}-12 v^{2}$ is fulfilled. If one changes in this solution $t$ by $-t$ and $x$ by $-x$, the new solution of the system (4) obviously describes the fusion of the soliton (3.32) and the wave (3.35) into one wave (3.21). Analogously, at $\kappa=-1$ our solution describes the decay of the wave (3.26) into the soliton (3.33) and the wave (3.38). Accoring to (3.8) we have in this case $\tau_{N}=4 \mu^{2}-12 v^{2}$, and by virtue of (3.10) the inequality $\left(4 \mu^{2}-12 v^{2}-\tau_{1}\right) \mu>0$ should hold. Finally, based on (3.40) we get the 
inequality $\tau_{1}<16 \mu^{2}-12 v^{2}$. Hence it follows that at $\mu>0$ the condition $\tau_{1}<4 \mu^{2}$ $-12 v^{2}$ is fulfilled and at $\mu<0$ the condition $4 \mu^{2}-12 v^{2}<\tau_{1}<16 \mu^{2}-12 v^{2}$ should hold. If now in this solution $t$ is changed by $-t$ and $x$ by $-x$, the new solution of the system (4) describes the fusion of the soliton (3.33) and the wave (3.38) into one wave (3.26). It is to be mentioned that at $\kappa=1$ and $\mu^{2}-3 v^{2}<0$ the equality $\tau_{1}=0$ is possible. Consequently, the wave (3.35) executes in this case an oscillatory motion in the finite limits. An analogous phenomenon may appear at $\kappa=-1$ as well if $4 \mu^{2}$ $-3 v^{2}>0$.

In conclusion, we should like to note that assuming $v=0$ in all the formulas of this paper, we get such solutions of the system (4) that the function $u$ is independent of $y$ and the function $\varphi$ admits the representation $\varphi=w \exp (-i E y)$, where the function $w$ is also independent of $y$ and $E$ is a certain constant. Hence it follows that the functions $u$ and $w$ satisfy the system of equations [6]

$$
\begin{gathered}
\frac{\partial u}{\partial t}+6 u \frac{\partial u}{\partial x}+\frac{\partial^{3} u}{\partial x^{3}}+8 \kappa \frac{\partial}{\partial x}|w|^{2}=0 \\
\frac{\partial^{2} w}{\partial x^{2}}+u w=E w
\end{gathered}
$$

Thus, the above assertions concerning the solutions of the system (4) obtained here can be made, with certain provisoes, also for the solutions of the system $(*)$.

\section{References}

1. Mel'nikov, V.K.: On equations for wave interactions. Lett. Math. Phys. 7, 129 (1983)

2. Mel'nikov, V.K.: A direct method for deriving a multi-soliton solution for the problem of interaction of waves on the $x, y$ plane. Commun. Math. Phys. 112, 639 (1987)

3. Mel'nikov, V.K.: Reflection of waves in nonlinear integrable systems. J. Math. Phys. 28, 2603 (1987)

4. Satsuma, Y.: $N$-soliton solution of the two-dimensional Kortweg-de Vries equation. J. Phys. Soc. Jpn. 40, 286 (1976)

5. Kadomtsev, B.B., Petiashvily, V.I.: Stability of solitary waves in weakly dispersing mediums. Dokl. Akad. Nauk SSSR 192, 753 (1970)

6. Zakharov, V.E., Kuznetsov, E.A.: Multi-scale expansions in the theory of systems integrable by inverse scattering transform. Physica 18D, 455 (1986)

Communicated by H. Araki

Received May 19, 1988 